\title{
IMMUNOCHEMICAL METHODS IN HEALTH RISK ASSESSMENT: CROSS REACTIVITY OF ANTIBODIES AGAINST MYCOTOXIN DEOXYNIVALENOL WITH DEOXYNIVALENOL-3-GLUCOSIDE
}

\author{
Jiří Ruprich, Vladimír Ostrý \\ National Institute of Public Health in Prague, Centre for the Hygiene of Food Chains in Brno, Czech Republic
}

\section{SUMMARY}

Co-occurrence of deoxynivalenol (DON) with other DON derivatives/metabolites and other Fusarium toxins, including zearalenone, nivalenol and as well as other mycotoxins (e.g. fumonisins) is frequently observed in food and feed. DON-3B-glucopyranoside (DON-3-glucoside) was described as detoxification product of DON in wheat. This mycotoxin conjugate was observed in maize, barley, malt, beer and wort. Digestion of this conjugate in intestine is still unclear but due to possibility to release DON after hydrolysis is considered as potential masked mycotoxin. DON is analytically quantified by various methods and also with immunochemical methods. There is no available information about specificity of anti-DON antibodies used in commercial ELISA kits with DON-3-glucoside.

Preliminary testing of anti-DON monoclonal antibodies used in ELISA kits RIDASCREENDON (R-Biopharm AG, Germany) approved a hypothesis that these antibodies have high relative cross reactivity with DON-3-glucoside. In two repeated tests cross reaction 82 and $98 \%$ were observed. Analytical results produced by these ELISA kits can be interpreted as an approximate sum of both mycotoxins. Described cross reactivity can lead to overestimating of DON concentration.

Over these cross reactions immunochemical methods are mentioned still valuable for quantitative screening and even for an initial exposure assessment in situations when there are practical or economical reasons not to use another analytical method with a reasonable low limit of quantification (<50 ppb).

Key words: mycotoxins, deoxynivalenol, deoxynivalenol-3-glucoside, immunoassay, antibodies, specificity

Address for correspondence: J. Ruprich, NIPH, Palackeho 3a, 61242, Brno, Czech Republic. E-mail: jruprich@chpr.szu.cz

\section{INTRODUCTION}

Immunochemical methods are frequently used for qualitative, semiquantitative and also quantitative measurements of many chemical substances with high biological impact. Between immunochemical methods also competitive enzyme immunoassays (ELISA) are well known and often used in a practice. These assays are used also for quantitative analyses of mycotoxins which can contaminate various feeds and foods.

Mycotoxin deoxynivalenol (CAS Number: 51481-10-8, Trihydroxy-12,13-epoxytrichothec-9-en-8-one, Rd toxin, DON, vomitoxin) (Fig.1) which belongs into a big group of trichothecene mycotoxins is produced by fungi from the genus Fusarium. DON is commonly detected at the ppm level.

The toxin is common in cereals and grains, particularly in wheat, barley and maize (1). Via these raw agriculture commodities DON can contaminate many different foods. Cereal foods and beer seems to be the most important groups for human dietary exposure assessment. In domestic animals, following absorption, DON is rapidly metabolised by de-epoxidation and glucuronidation, yielding less toxic products (3). Transfer of DON

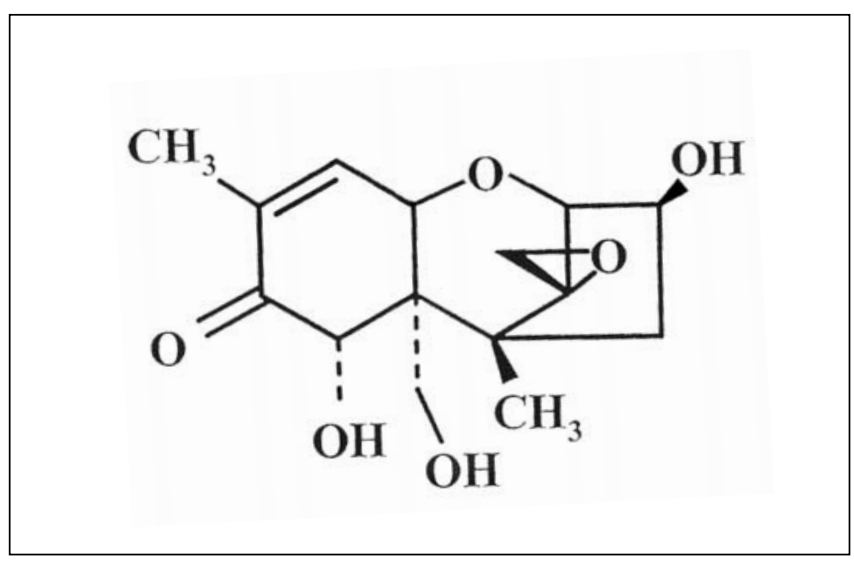

Fig. 1. Mycotoxin deoxynivalenol (DON, vomitoxin).

and its metabolites into edible tissues, milk and eggs is very low. Thus, products of animal origin do not contribute significantly to human exposure. Products of plant origin play substantial role in human exposure. Considering the year-to-year variability of the contamination of cereals and grains with DON in Europe, an 
exposure of consumers close to the TDI of $1 \mu \mathrm{g} / \mathrm{kg}$ b.w./day is possible (1).

Acute exposure to extremely high DON $[\geq 27 \mathrm{mg} / \mathrm{kg}$ body weight (b.w.) doses] is required to cause mortality or marked tissue injury in experimental animals. In contrast, acute exposure to relatively low doses ( $\geq 50 \mu \mathrm{g} / \mathrm{kg}$ b.w.) can cause vomiting in pigs, the most sensitive species. This corresponds to human food poisoning outbreaks with nausea, diarrhea and vomiting as primary symptoms that were associated with Fusarium-infested cereals. The most common effects of prolonged dietary exposure of experimental animals to DON are decreased weight gain, anorexia, decreased nutritional efficiency and altered immune function with species differences again being apparent (2). There is no evidence for teratogenicity and genotoxicity of DON and its metabolites, neither in laboratory animal species nor in target animals (1).

Co-occurrence of DON with other DON derivatives/metabolites and other Fusarium toxins, including zearalenone, nivalenol and as well as other mycotoxins (e.g. fumonisins) is frequently observed. Both 3- and 15-acetyl DON, which sometimes co-occur in smaller amounts cereal grains, are equivalently or less toxic than DON based on LD50 values in mice and are thus unlikely to pose any additional risk (2). Plants infected by toxigenic fungi, including cereals, similarly to animals, can partly convert some mycotoxins.

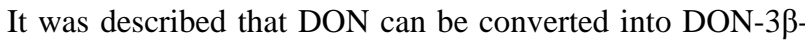
glucopyranoside (DON-3-glucoside, D3G) (Fig. 2) in wheat as detoxification product (4). Firstly was DON-3-glucoside described as a plant metabolite of DON in 1986 (5).

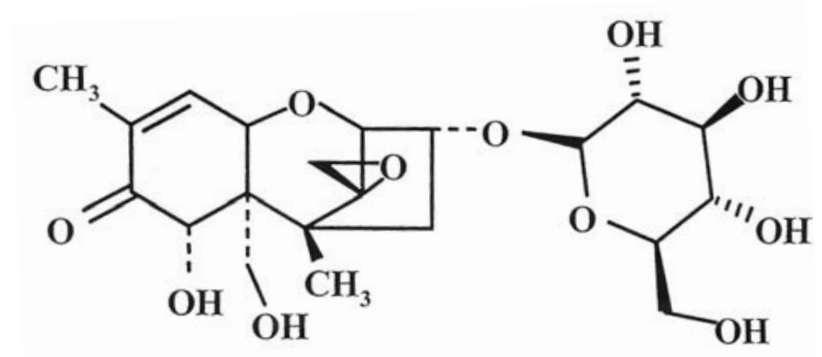

Fig. 2. Mycotoxin DON-3 $\beta$-glucopyranoside (DON-3-glucoside, $D 3 G)$.

Some authors suggested to recognize DON-3-glucoside as a potential masked mycotoxin because it can escape routine detection methods and contribute to the total amount of DON in particular foods or feeds $(5,6)$. The term masked mycotoxin was used in year 1990 by Gareis et al. (7) who described similar situation for zearalenone-4-O-glucoside which is able to be cleaved during digestion in swine. The cleavage ability for DON-3-glucoside (hydrolysis) was studied recently (6). DON-3-glucoside was found in naturally contaminated wheat up to $12 \%$ of the DON value and in naturally contaminated maize even up to $50 \%$ of the DON value. Therefore possible additional mycotoxin load was not considered as negligible. DON-3-glucoside showed high stability to acidic conditions down to a $\mathrm{pH}$ value of 0,7 therefore its hydrolysis in stomach is unlikely. Human and plant $\beta$-glu- cosidases had no hydrolytic effect. Cellulases could partially hydrolyse DON-3-glucoside. Moreover it was also demonstrated that several bacterial strains such as Enterococcus durans, Enterococcus mundtii and Lactobacilus plantarum had a capacity to hydrolyse DON-3-glucoside and release DON. There is still no clear evidence that these facts have substantial toxicological relevance in animals and humans.

DON-3-glucoside and also DON-15-glucoside were chemically synthesized and characterized $(5,8)$ by liquid chromatography-tandem mass spectrometry (LC-MS/MS). Now both of them are commercially available as analytical standards from Biopure Referenzsubstanzen GmbH, Austria. It opened possibility for other research teams to continue with related research on DON-glycosides.

Transfer of Fusarium mycotoxins including DON and DON-3glucoside from field barley through malt to beer has been studied recently $(10,11)$. Results of this study anticipate a hypothesis that beer can contain DON-3-glucoside in concentration values which are higher than for DON and therefore it can be important for an exposure assessment in groups of beer consumers.

New results for DON and DON-3-glucoside in beer, beside previously published analytical results for DON in beer (15), elicitated discussion about acceptability of analytical results from available ELISA methods for health risk assessment. These commercially available ELISA kits (e.g. RIDASCREEN ${ }^{\circledR}$ DON, R-Biopharm AG, Germany) are designed for quantitative analyses of DON in cereals, malt, feed, beer and wort (12). Due to relatively low limit of quantification of these ELISA methods and relatively low cost per one analysis some laboratories use these results for an estimate of low concentrations of DON in foods of cereal origin. These results then may serve as a data source for point or probabilistic modeling of exposure doses for population groups $(13,14)$.

A decision making when analytical results from ELISA methods can be used for health risk assessment depends on their qualitative parameters involving types of antibodies, their affinity and specificity. Policy on characterization of antibodies used in immunoassays for mycotoxins and phycotoxins were already suggested (16).

Based on above mentioned information we decided to preliminary test the specificity of anti-DON monoclonal antibodies used in ELISA kits RIDASCREEN ${ }^{\circledR}$ DON (R-Biopharm AG, Germany) because these are frequently used in central European countries for many years. Knowledge of specificity is needed to decrease uncertainty connected with interpretation of analytical results. Basic experimental question was to describe a cross reaction between anti-DON antibodies used in mentioned commercial kits with DON-3-glucoside and to estimate how this specificity can influence an interpretation of analytical results.

\section{METHODS}

\section{Work Protocol and Calculation of Specificity}

Preliminary testing of specificity of anti-DON monoclonal antibodies was based on the work protocol recommended by ELISA kit producers (12). For the purpose of the experiment we used various concentrations of DON and DON-3-glucoside 
instead of food samples (external standards). From results it was possible to calculate relative cross-reactivity (CR, specificity) in $\%$ as followed:

$$
\mathrm{CR}(\%)=\mathrm{C}_{\mathrm{DON}} / \mathrm{C}_{\mathrm{DON}-3 \text {-glucoside }} * 100 \text {, where }
$$

$\mathrm{C}_{\mathrm{DON}}$ is concentration of DON needed for $50 \%$ reduction of absorbance of the zero control standard

$\mathrm{C}_{\text {DON-3-glucoside }}$ is concentration of DON-3-glucoside needed for $50 \%$ reduction of absorbance of the zero control standard.

\section{Standards}

DON standard (Sigma USA), Cat. No. D-0156; DON-3-glucoside standard (Biopure Referenzsubstanzen GmbH, Austria), BRM Cat. No. S02012.

\section{ELISA Kits}

RIDASCREEN ${ }^{\circledR}$ DON (R-Biopharm AG, Germany), Lot: 05017, Exp. 2008-05. We used test procedure and reagents contained or recommended in RIDASCREEN ${ }^{\circledR}$ DON ELISA kits. For each internal standard/sample (external standard) we used 3 wells in micro well strips.

\section{Equipment}

Reader - photometer MRX 1200, DYNATECH Laboratories.

\section{RESULTS AND DISCUSSION}

A simple experiment has been designed to model a daily routine in laboratory when DON is quantified by ELISA kits. An experiment was repeated twice in independent days. External standard of DON and DON-3-glucoside was used in 12 dilutions. In the first test the range was $2.5 \mathrm{ppb}-5,000 \mathrm{ppb}$, in the second test the range was $0.625 \mathrm{ppb}-1,250 \mathrm{ppb}$. Fig. 3 and 4 show curves which we obtained when we compared reduction of absorbance with external standard of DON and DON-3-glucoside. Concen-

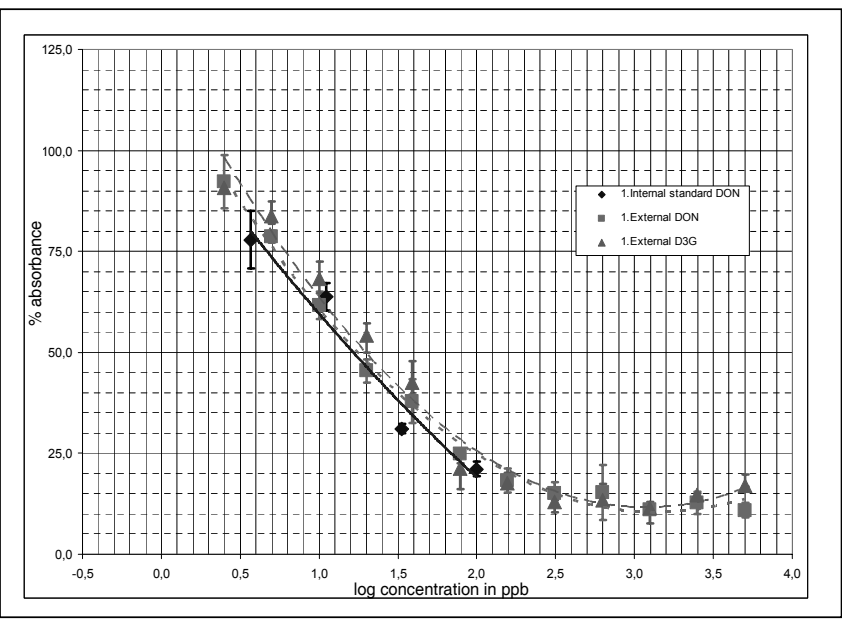

Fig. 3. Comparison of the cross reactivity of anti-DON monoclonal antibodies used in ELISA kits RIDASCREEN ${ }^{\circledR} D O N$ (1st test, range 2.5-5,000 ppb).

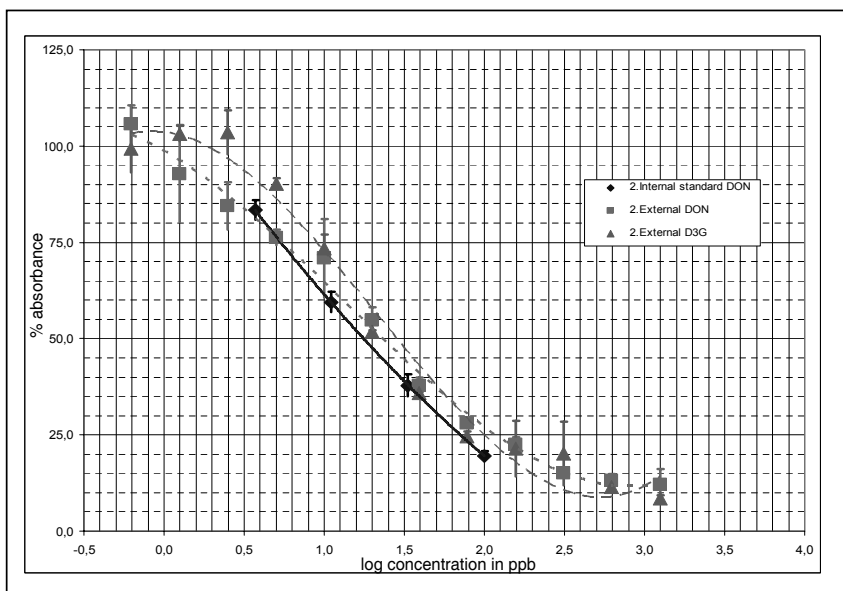

Fig. 4. Comparison of a cross reactivity of anti-DON monoclonal antibodies used in ELISA kits RIDASCREEN囚DON (2nd test, range $0.625-1,250 \mathrm{ppb}$ ).

tration points in figure are characterized by response in \% of absorbance (mean $\pm 1 \mathrm{SD}$ ). Curves were calculated with usage of polynomial functions of the second and the third degree.

Calculation of numeric values of the cross reactivity of used antiDON monoclonal antibodies depends on variability and uncertainty of input data and used regression methods. For the purpose of our testing we finally decided to use only data within a range of internal DON standards (3.7 - $100 \mathrm{ppb}$ ) provided in every ELISA kit and use simple linear regression within this range. Therefore only data for concentration of external standards in range $5-78 \mathrm{ppb}$ (5 external standard simulating samples) of DON and DON-3-glucoside were used for a calculation. To decrease possible bias given by a low number of repetitions of tests (two) we decided to calculate only relative cross reactivity of antibodies between external standards. External DON standard was used as reference value (100\%) and external DON-3-glycoside standard was compared with it. Following Table 1 summarizes results of this work

\section{CONCLUSIONS}

Preliminary testing of anti-DON monoclonal antibodies used in ELISA kits RIDASCREEN ${ }^{\circledR}$ DON (R-Biopharm AG, Germany) approved a hypothesis that these antibodies have high relative cross reactivity with DON-3-glucoside. In two repeated tests cross reaction 82 and $98 \%$ were observed. This is important for interpretation of analytical results because DON-3-glucoside is expected as a detoxification product of DON in foods and feeds of cereal origin. Analytical results produced by these ELISA then kits can be interpreted as an approximate sum of both mycotoxins. Described cross reactivity can lead to overestimating of DON concentration. Over these cross reactions immunochemical methods are mentioned still valuable for quantitative screening and even for an initial exposure assessment in situations when there are practical or economical reasons not to use another analytical method with a reasonable low limit of quantification $(<50 \mathrm{ppb})$. 
Table 1. Relative cross reactions of anti-DON monoclonal antibodies used in ELISA kits RIDASCREEN®DON with mycotoxin DON-3-glycoside

\begin{tabular}{|l|c|c|c|c|c|}
\hline Analytes in tests & $\begin{array}{c}\text { Absorbance } \\
\text { (\% of zero } \\
\text { standard) }\end{array}$ & Concentration $(\mathbf{p p b})$ & $\begin{array}{c}\text { Cross } \\
\text { reactivity } \\
(\%)\end{array}$ & $\begin{array}{c}\text { Regression } \\
\text { method }\end{array}$ & $\begin{array}{c}\text { Regression } \\
\text { coefficient } \\
\left(\mathbf{r}^{2}\right)\end{array}$ \\
\hline 1. External DON & 50 & 19.5 & 100 & linear & 0.98 \\
\hline 1. External DON-3-glucoside & 50 & 23.8 & 82 & linear & 0.99 \\
\hline 2. External DON & 50 & 23.9 & 100 & linear & 0.98 \\
\hline 2. External DON-3-glucoside & 50 & 24.5 & 98 & linear & 0.99 \\
\hline
\end{tabular}

\section{REFERENCES}

1. EFSA 2004. Opinion of the scientific panel on contaminants in the food chain on a request from the commission related to deoxynivalenol (DON) as undesirable substance in animal feed. EFSA J. 2004;73:1-42.

2. Pestka JJ. Deoxynivalenol: toxicity, mechanisms and animal health risks. Anim Feed Sci Technol. 2007 Oct;137(3-4):283-98.

3. Wu X, Murphy P, Cunnick J, Hendrich S. Synthesis and characterization of deoxynivalenol glucuronide: its comparative immunotoxicity with deoxynivalenol. Food Chem Toxicol. 2007 Oct;45(10):1846-55.

4. Lemmens M, Scholz U, Berthiller F, Dall'Asta C, Koutnik A, Schuhmacher R, et al. The ability to detoxify the mycotoxin deoxynivalenol colocalizes with a major quantitative trait locus for Fusarium head blight resistance in wheat. Mol Plant Microbe Interact. 2005 Dec;18(12):131824.

5. Berthiller F, Dall'Asta C, Schuhmacher R, Lemmens M, Adam G, Krska R. Determination of DON-3-Glucoside in artificially and naturally contaminated wheat with LC-MS/MS. In: Degen GH, et al. 27th. Mycotoxin Workshop; 2005 Jun 13-15; Dortmund. Dortmund: Society for Mycotoxin Research; 2005. p. 101.

6. Berthiller F, Doming KJ, Kneifel W, Juge N, Knasmüller S, Adam G, et al. Hydrolysis of deoxynivalenol-3-O-glucoside. In: 29th Mycotoxin Workshop; 2007 May 14-16; Stuttgart - Fellbach. Fellbach: Society for Mycotoxin research; 2007. p. 43.

7. Gareis M, Bauer J, Thiem J, Plank G, Grabley S, Gedek B. Cleavage of zearalenone-glycoside, a "masked” mycotoxin, during digestion in swine. Zentralbl Veterinarmed B. 1990 May;37(3):236-40.

8. Dall'Asta C, Berthiller F, Schuhmacher R, Adam G, Lemmens M, Krska R. DON-Glycosides: characterization of synthesis products and screening for their occurrence in DON-treated wheat samples. Mycotoxin Res. 2005;21(2):123-7.
9. Sewald N, von Gleissenthall JL, Schuster M, Muller G, Aplin RT. Structure elucidation of a plant metabolite of 4-desoxynivalenol. Tetrahedron Asymmetry. 1992 Jul;3(7):953-60.

10. Hajslová J, Lancová K, Kostelanská M, Zachariasová M, Poustka J. Fusarium mycotoxins: transfer from field barley through malt to beer. In: XIIth International IUPAC Symposium on Mycotoxins and Phycotoxins; 2007 May 21-25; Istanbul. Istanbul: 2007.

11. Lancová K, Krplová A, Hajslová J, Sachambula L, Vanova M. Fusarium mycotoxins: transfer from field barley through malt to beer. In: XIIth International IUPAC Symposium on Mycotoxins and Phycotoxins; 2007 May 21-25; Istanbul. Istanbul: 2007.

12. RIDASCREEN ${ }^{\circledR}$ DON: guide for a competitive enzyme immunosorbent assay for quantitative analysis of deoxynivalenol in cereals, malt, feed, beer and wort. 05-07-27. Darmstadt: R-Biopharm AG; 2007.

13. Ostry V, Ruprich J, Skarková J. The dietary exposure od deoxynivalenol in the Czech Republic. In: 28th Mycotoxin Workshop; 2006 May 29-31; Bydgoszcz. Bydgoszcz: Society for Mycotoxin research; 2006. p. 118.

14. Ruprich J, Ostry V, Skarková J. Probabilistic assessment of exposure doses to mycotoxins: case of DON in foodstuffs. In: 29th Mycotoxin Workshop; 2007 May 14-16; Stuttgart - Fellbach. Fellbach: Society for Mycotoxin research; 2007. p. 131.

15. Ruprich J, Ostrý V. Determination of the mycotoxin deoxynivalenol in beer by commercial ELISA tests and estimation of the exposure dose from beer for the population in the Czech Republic. Cent Eur J Public Health. 1995 Nov;3(4):224-9.

16. Fremy JM, Usleber E. Policy on characterization of antibodies used in immunochemical methods of analysis for mycotoxins and phycotoxins. J AOAC Int. 2003 Jul-Aug;86(4):868-71.

Received and accepted October 25, 2007 